\title{
Recurrent Acute Diverticulitis: When to Operate?
}

\author{
Hassan Al Harakeh $^{\mathrm{a}}$ Abhilash J. Paily ${ }^{\mathrm{b}}$ Samer Doughan ${ }^{\mathrm{a}}$ Irshad Shaikh $^{\mathrm{b}}$ \\ ${ }^{a}$ American University of Beirut - Medical Center, Beirut, Lebanon; ${ }^{b}$ Norfolk and Norwich University Hospital, \\ Norwich, UK
}

\author{
Keywords \\ Diverticular disease · Recurrent diverticulitis · Surgery for \\ diverticulitis
}

\begin{abstract}
Objective: Recurrent acute diverticulitis carries a major burden to any form of health care. Patients present repeatedly to medical centers with a multitude of symptoms and may require different modalities of treatment with significant morbidities and impact on quality of life. Methods: We therefore wanted to identify factors that would imply the need and time of surgery versus conservative management. The literature was thoroughly searched for major studies tackling this topic. Furthermore, studies reporting on decision making based on quality of life were included. Risks of developing recurrent diverticulitis and the potential need of surgery were identified. Relevant surgical details that would decrease recurrence were also denoted. Results: Surgery has been the mainstay of treatment for quite some time. However, the paradigms of treatment have changed over the last few years, especially when long-term population studies confirmed that not all patients require surgical treatment with its associated risk of morbidity. Conclusion: Treatment
\end{abstract}

now has to be patient-tailored with special attention to the subgroup of high-risk patients. These patients must be adequately selected, identifying the impact of the disease on the quality of life and weighing in the risks of the surgical intervention.

(C) 2018 S. Karger AG, Basel

\section{Introduction}

Recurrent diverticulitis can occur after a nonsurgically managed acute attack. Rarely, it can also present in patients after resection for sigmoid diverticulitis. Recurrent attacks of acute diverticulitis are seen in $20-35 \%$ of patients presenting with the first attack of acute diverticulitis [1-10]. This occurs despite complete remission and is a major economic burden amongst diseases of the gut. Additionally, 36\% of people have ongoing abdominal symptoms after the first episode of diverticulitis [11-13]. As the incidence of diverticulitis increases, one would infer that recurrent attacks would begin to increase in frequency. Mechanisms responsible for these attacks are not clear. There is insufficient literature to determine risk factors responsible for recurrence after a primary attack in a

\section{KARGER}

(c) 2018 S. Karger AG, Basel

E-Mail karger@karger.com

www.karger.com/iid
Hassan Al Harakeh

American University of Beirut - Medical Center

Beirut (Lebanon)

E-Mail ha94aub.edu.lb 
patient who has never undergone surgical intervention for acute diverticulitis. Hupfeld et al. [14] showed that diverticular abscess formation and younger people were at a higher risk of recurrence. Similarly, Anaya and Flum [2] noted younger patients ( $<50$ years) had a higher rate of not only recurrence ( 27 vs. $17 \%$ ), but also emergency colectomy/colostomy ( 7.5 vs. $5 \%$ ). However, Katz et al. [15] disputed the assertion of increased severity of diverticulitis in young patients and found in a metanalysis of nearly 5,000 patients aged $40-50$ years that diverticular disease in this age group was not more severe than that in the elderly. Nonetheless, it was found that the frequency of representation was higher in the young and male population (RR 1.70, 95\% CI 1.31-2.21) [15].

Recurrent admissions were highest in the first year of diagnosis. The risk of recurrence appeared to increase after each recurrence. However, a left colon with more than $5 \mathrm{~cm}$ of inflammation, presence of multiple and pan-colonic diverticula, and immunosuppression carried a medium risk only. Poletti et al. [1] followed up patients for 18 months and found that $32 \%$ of patients presented with recurrent diverticulitis. Computed tomography (CT) scan signs of evidence of gas pocket and abscess were predictors of recurrence. Age and sex were not significantly associated with the risk of recurrence. Hall et al. [7] in a study of 672 patients showed that family history of diverticulitis, long segment of colon involvement, and the presence of retroperitoneal abscess were predictors of failure of nonoperative management. However, this study also clarified that in 5 years of follow up 3.9\% presented with complicated diverticular disease including fistulae, abscess, or frank perforation. Similarly, Eglinton et al. [5] noted a $5 \%$ rate of complicated presentations after conservative management in a study of more than 300 patients followed up for a median period of 101 months. Interestingly, right-sided colonic diverticulitis was not associated with recurrence (HR 0.27, 95\% CI 0.09-0.86). Prophylactic use of mesalamine has been explored and may reduce the frequency of recurrences [14].

Historically, surgery was advised after two attacks of uncomplicated diverticulitis and after one attack in patients younger than 40 years [16]. This has been recently challenged by a new approach to individualize treatment depending on patient immunity status, development of chronic pain, frequency of attacks, and complications the patients develop, and after some new studies that reflected on the rates of events of patients with history of acute diverticulitis. For this reason, we thought of embarking on this review to identify relevant data in support of indication, timing, and method of surgery.
Table 1. Risk factors for diverticulitis

\begin{tabular}{|c|c|}
\hline Factor & Positive association \\
\hline Low dietary fiber & + \\
\hline Red meats & + \\
\hline Overweight or obesity & + \\
\hline Sedentary lifesytyle & + \\
\hline Smoking (>40 packyears) & + \\
\hline Seeds, nuts, and popcorn & - \\
\hline Caffeine & - \\
\hline Alcohol & - \\
\hline NSAIDs, steroids, and opiates & + \\
\hline Vitamin D and statins & -- \\
\hline
\end{tabular}

\section{Pathophysiology}

Diverticulosis is defined as the occurrence of small out-pouchings or protrusions in intestinal walls. These pockets, called diverticula, generally remain asymptomatic. However, they can become symptomatic and when they do, the condition is defined as diverticular disease which comprises a number of disorders including bleeding, diverticulitis, or segmental colitis. Diverticulitis occurs when the diverticula become inflamed. Diverticular disease presents a major economic burden in Western societies.

Typical diverticula are false or pseudo-diverticula that are composed of mucosa and submucosa herniating through the muscularis layer and covered only by serosa. This commonly occurs at the relatively weak points where the vasa recta penetrate the muscularis layer to perfuse the submucosa and mucosa.

Numerous risk factors have been associated with diverticular disease and many were studied (Table 1). In a prospective cohort that assessed the risk of diverticulitis in over 51,000 men aged 40-75 years, low dietary fiber, high intake of red meat, being overweight or obese, sedentary lifestyle, and smoking ( $\geq 40$ pack-years) were independently associated with an increased risk of diverticulitis [17]. In contrast to previous perception, seeds, nuts, and popcorn are not associated with increased risk of diverticulosis or diverticular disease, nor were caffeine and alcohol [18]. Smoking on the other hand is not only associated with diverticular disease but also appears to confer an increased risk of perforation and abscess formation [19]. Drugs also play a significant role in the pathogenesis of diverticular disease. While nonsteroidal anti-inflam- 
matory drugs, steroids, and opiates are associated with increased risk of diverticulitis and bleeding, vitamin D and statins bestow a protective effect [19-21].

\section{Diverticulitis}

With advanced inflammation, microscopic or macroscopic perforation of a diverticulum can happen due to focal necrosis and this divides diverticulitis into two categories: uncomplicated and complicated.

Uncomplicated diverticulitis is a localized inflammation of the diverticulum, while complicated diverticulitis is advanced inflammation concomitant with bleeding or the formation of a phlegmon, abscess, or fistula or associated with obstruction or purulent or fecal peritonitis. Diverticulitis happens in approximately $4 \%$ of patients with diverticulosis [22].

Because the sigmoid colon is most frequently affected, diverticulitis usually presents as left lower-quadrant abdominal constant pain. When the sigmoid is redundant it may cause suprapubic or even right lower-quadrant pain. Asian populations have a relatively higher incidence of cecal diverticulitis. On the other hand, laboratory tests are supportive in the diagnosis but are not sensitive or specific.

\section{Diagnosis}

CT scan of the abdomen with a sensitivity and specificity for the diagnosis of acute diverticulitis of 94 and 99 percent, respectively, is usually used to confirm the diagnosis. However, colonoscopy should be avoided in the acute setting due to the risk of perforation, and unless the patient has had a recent colonoscopy, it should be performed at least 6 weeks after remission of the diverticulitis to exclude the possibility of an underlying colorectal cancer [23].

In addition to being widely available, inexpensive, and avoiding radiation exposure, high-resolution ultrasound has comparable sensitivity and specificity for acute diverticulitis as compared with abdominal CT scan. Nonetheless, abdominal ultrasound is operator dependent and cannot exclude other causes of abdominal pain [23].

\section{Recurrent Diverticulitis: Risk Factors}

Readmission rate for recurrent diverticulitis ranges from 9 to $25 \%$ [6, 8, 11-13] (Table 2). After a follow-up of 4 years, El Sayed et al. [6], in an English study of over

Recurrent Acute Diverticulitis: When to Operate?
65,000 patients managed nonoperatively for their first episode of diverticulitis, found the recurrence rate to be around $11.2 \%$. Emergency and elective colectomy rates were 0.9 and $0.75 \%$, respectively. Female sex, young age, smoking, obesity, and complicated initial disease were risk factors for readmission and emergency surgery.

On the other hand, around 14,000 Canadian patients who were treated nonoperatively as well were followed up for almost 4 years in a study by Li et al [8]. The readmission rate was $9 \%$ while emergency surgery and elective colectomy rates were 1.9 and $1.7 \%$, respectively.

Risk factors associated with recurrence were younger age and the persistence of postoperative pain. In other studies, the likelihood of recurrence was affected by the severity of the initial episode and not by age at onset [9].

Conservative therapy is successful in keeping 30\% of these patients completely asymptomatic after the first attack. However, in a population-based study that reviewed the records of 3,222 patients with acute diverticulitis with a 10-year follow-up, recurrent diverticulitis occurred after the index and second diverticulitis episodes in 22 and $55 \%$ of patients, respectively. Female gender and younger age were associated with higher recurrence rate $[4,7]$.

Furthermore, recurrences do not infer higher risk of complications compared to first episodes [9]. After surgery, new diverticula develop in the remaining colon in around $15 \%$ and roughly $2-11 \%$ will require repeat surgery $[3,10]$. Historically, recurrence of diverticulitis after surgery has been in the range of $7-11 \%$ with an estimated risk of recurrence over a 15-year period of $16 \%$ [3]. Recent data from several observational studies revealed recurrence rates of $10-35 \%$ after a first episode of uncomplicated diverticulitis $[2,5,7]$.

\section{Treatment}

Prophylactic colectomy was advocated after two attacks of uncomplicated diverticulitis and after one attack in patients younger than 40 years [16]. The main reasons for offering surgical resection of recurrent diverticulitis are (1) to prevent further attacks and improve quality of life, (2) to prevent potential complications such as colovescial fistulae or colovaginal fistulae, and (3) to prevent the potential risk of an emergency procedure resulting in colostomy formation. However, the advent of high-resolution CT scans to determine the severity of inflammation and the introduction of less invasive interventions for draining the infectious process have changed the approach a surgeon now takes. There is a move towards se- 
Table 2. Pearls of recurrent diverticulitis

\begin{tabular}{|c|c|}
\hline Diverticulitis occurs in $4 \%$ of patients with diverticulosis & {$[22]$} \\
\hline Recurrent diverticulitis Rate after primary attack treated conservatively: $11-35 \%$ & {$[1-10,13]$} \\
\hline Readmission rate for recurrent diverticulitis $9-25 \%$ & {$[6,8,11,12,38]$} \\
\hline Recurrent diverticulitis after surgery $7-16 \%$ & {$[3,38]$} \\
\hline $36 \%$ have ongoing abdominal symptoms after the first episode of diverticulitis & [11-13] \\
\hline Persistence of post-operative symptoms is a risk factor for recurrent diverticulitis & {$[8]$} \\
\hline Severity of first episode is a risk factor for recurrent diverticulitis & {$[8]$} \\
\hline CT scan evidence of gas pockets and abscess formation is a risk factor for recurrence & {$[1,14]$} \\
\hline Younger age is a risk factor for recurrence & {$[2,4,6-8,14,15]$} \\
\hline Younger age is a risk factor for emergency colectomy/colostomy & {$[2,6]$} \\
\hline Diverticular disease in younger age is not more severe & {$[15]$} \\
\hline Male gender is a risk for recurrent diverticulitis & {$[15]$} \\
\hline Female gender is a risk for recurrent diverticulitis & {$[4,6,7]$} \\
\hline Family history of diverticulitis is a risk factor for failure of non-operative management & {$[7]$} \\
\hline Long segment of involved colon is a risk factor for failure of non-operative management & {$[7]$} \\
\hline Presence of retroperitoneal abscess is a risk factor for failure of non-operative management & {$[7]$} \\
\hline Complicated recurrence rate $3.9-5 \%$ & {$[5,7]$} \\
\hline Right-sided colonic diverticulitis not associated with recurrence & {$[5]$} \\
\hline $\begin{array}{l}\text { Surgery is recommended after } 2 \text { episodes of diverticulitis and after one episode in patients } \\
\text { younger than } 40 \text { years }\end{array}$ & {$[16]$} \\
\hline Emergency surgery for diverticulitis is $0.9-1.9 \%$ & {$[6,8]$} \\
\hline Elective colectomy for diverticulitis is $0.75-1.7 \%$ & {$[6,8]$} \\
\hline \multicolumn{2}{|l|}{ Likelihood of recurrent diverticulitis increases with number of recurrences } \\
\hline Recurrences do NOT infer higher risk of complications compared to the first episode & {$[9,30,39,40]$} \\
\hline $\begin{array}{l}\text { Elective resection carries a lower risk of persisting symptoms compared to conservative } \\
\text { management }\end{array}$ & {$[11,13,42]$} \\
\hline Overlap between post-diverticulitis symptoms and irritable bowel syndrome & {$[28]$} \\
\hline Risk of Hartmann's procedure after one diverticulitis attack is 1 in 2,000 & {$[30]$} \\
\hline 18 elective colonic resections are needed to prevent one colostomy & {$[30]$} \\
\hline $92 \%$ of recurrent cases were treated as outpatient & {$[41]$} \\
\hline $2 \%$ of recurrent cases ultimately needed surgery & {$[41]$} \\
\hline $90 \%$ of chronic smoldering diverticulitis benefit from surgery & {$[43]$} \\
\hline $77 \%$ of chronic smoldering diverticulitis had complete resolution of symptoms after surgery & {$[25]$} \\
\hline $\begin{array}{l}\text { Comparable complication rate between Hartmann's and Primary anastomosis with } \\
\text { stoma formation }\end{array}$ & {$[45,46]$} \\
\hline $\begin{array}{l}\text { Stoma reversal with primary anastomosis was higher than Hartmann's procedure in } \\
\text { grade III and IV Hinchey Diverticulitis }\end{array}$ & {$[45,46]$} \\
\hline Total sigmoidectomy is associated with lower recurrence & {$[3,47]$} \\
\hline
\end{tabular}


lective colectomy after one episode of uncomplicated diverticulitis on a background of recurrent acute diverticulitis. Quality of life is fast becoming the reason why patients choose to have, or not to have, elective sigmoid colectomy [11].

In 2006, the American Society of Colon and Rectal Surgeons suggested a tailored approach in which complaints about quality of life and the impact of multiple recurrences would aid patient decision making [24]. Even though resection reduces the occurrence of further episodes, the actual impact on quality of life is not clearly understood. There is no high-level evidence to support either conservative management or surgical treatment for recurrent diverticulitis. Some studies have shown a lower incidence of persisting symptoms after elective resection compared to conservative management (95 vs. $36 \%$, respectively) [13]. A meta-analysis by Andeweg et al. [13] in 2016 showed that patients who underwent resection had a better mean SF-36 score (73.4 vs. 58.2$)$ and a lower occurrence of chronic abdominal pain (11 vs. $38 \%$ ) compared to those who were managed conservatively. However, the studies included were mainly noncomparative cohort studies and had a high incidence of bias. There is a general reluctance amongst surgeons to offer resection based on uncertainties in differentiating functional bowel disorders from diverticulitis. A huge overlap of symptoms exists. Altered motility, change in gut microbial flora, low-grade inflammation, and visceral hypersensitivity have been attributed to persistent chronic abdominal pain after diverticulitis [25-27]. These mechanisms overlap in patients with irritable bowel syndrome. Some studies have postulated that diverticulitis could lead to irritable bowel syndrome [28]. As a result, it would be counterproductive for these patients to undergo surgical treatment. The DIRECT trial, however, appears to undermine this rationale.

van de Wall et al. [11] aimed to show, in the open-label randomized clinical DIRECT trial, which treatment modality had a better disease-specific and general quality of life measured by the Gastrointestinal Quality of Life Index (GIQLI) in patients presenting with either recurrent or persistent abdominal complaints after an episode of left-sided diverticulitis confirmed by CT, ultrasound, or endoscopy and followed for 6 months after a first attack of diverticulitis.

However, the trial was stopped prematurely because of difficulty in recruitment. Patients were recruited from 24 centers in the Netherlands. Out of 431 patients assessed for eligibility only 109 patients were included in the analysis. The main reasons for dropouts were patient prefer-

Recurrent Acute Diverticulitis: When to Operate? ence for type of management and patients not fitting the criteria for recurrent diverticulitis. Results from this trial showed a significantly better general and disease-specific quality of life following elective resection than with patients managed conservatively after multiple episodes of recurrent or ongoing chronic abdominal pain after the first attack of diverticulitis. They also suggest that persistent symptoms after one attack of diverticulitis were not caused by functional bowel disorders. On the downside, this study showed a $12 \%$ anastomotic leak rate and an $18 \%$ covering stoma formation rate which is much higher than the rates published in the literature. The authors concluded that there was significant difference of 14.2 points on the GIQLI score between surgical and conservative treatment.

Patient selection is very important. With the development of interventional radiology, it is reasonable to expect the requirement of elective resection of diverticulitis to decline [29]. Janes et al. [30] showed that the risk of Hartmann's resection after a solitary attack of diverticulitis is 1 in 2,000 patient-years of follow-up. About 18 elective colonic resections are required to prevent one colostomy.

Patients suffering recurrent episodes were deemed to have a $60 \%$ risk of acquiring complications after resection and were not likely to respond to medical therapy [11]. However, elective sigmoid colectomy for diverticulitis is controversial. The literature has shown that most of the complications occur during the first attack, implying that subsequent attacks will be of lower intensity. Therefore, it appears that elective resection to prevent future complications is unwarranted.

The intention of elective surgery has been to reduce emergency procedures, the need to create stomas, and to reduce the risk of complicated recurrence [31-37]. Conversely, this was defied in recent years by Andeweg et al. [38] who had an estimated risk of recurrence of $16 \%$ over 15 years; this translates to 1 of every 6 patients risking a recurrence after resection. Moreover, recurrent diverticulitis did not imply a higher rate of emergency surgeries. Ritz et al. [9], in their retrospective cohort of more than 1,300 patients, found that free perforation occurred in 25 , 12,6 , and $1 \%$ with their first, second, third, and thereafter episodes, respectively.

Others have even found that complication and colostomy rates were not higher after the fourth recurrence episodes as compared to surgery after the first episode $[30,39,40]$. In fact, in a study of 110 patients with acute diverticulitis, $18 \%$ had one recurrence and $6 \%$ had two recurrences over a period of 10.5 years; those who re- 
curred were treated as outpatients in $92 \%$ of cases and ultimately only $2 \%$ needed surgery [41].

On the other hand, Morris et al. [42], in their systematic review of 80 studies, found that chronic pain develops in $20-35 \%$ of patients treated nonoperatively compared with $5-25 \%$ of patients treated surgically. Many other authors have noted in their practice that there is a group who presents with atypical symptoms or chronic symptoms. These features do not fit in with acute diverticulitis as inflammatory markers are often raised. It is difficult to differentiate this entity from irritable bowel syndrome or chronic pain syndromes. There is another entity called chronic smoldering diverticulitis. These patients do not have complicated diverticulitis yet suffer severely with symptoms. Boostrom et al. [43] showed that operating on chronic smoldering diverticulitis can benefit up to $90 \%$ of patients while Horgan et al. [25] found that $77 \%$ of patients with smoldering diverticulitis achieved complete resolution of their chronic pain after sigmoid resection. Operating on smoldering diverticulitis is for treatment of symptoms and not to reduce the recurrence rate, unlike the general trend where patients undergo surgery to prevent recurrent diverticulitis after nonoperative treatment of an episode of complicated diverticulitis. Furthermore, complicated diverticulitis carries a higher morbidity and mortality should a recurrent episode of diverticulitis occur [44].

Many randomized trials have studied the use of mesalamine in the treatment of diverticulitis. Results have been variable [31, 32]. Carter et al. [33], in a 2017 Cochrane systematic review and meta-analysis of seven randomized trials, found no evidence of the benefit of mesalamine for the prevention of recurrent diverticulitis. In another study, 117 patients with acute diverticulitis were randomized to received placebo, mesalamine, or mesalamine plus a probiotic for 3 months and were followed up for 9 months. Mesalamine appeared to have lower symptom scores compared with patients who received placebo [31]. It is postulated that mesalamine may, therefore, have a role in conservatively managing patients with recurrent diverticulitis in the future.

\section{Surgical Approach}

Elective surgery for diverticular disease is generally delayed at least 6 weeks after the episode so that the inflammation and infection extensively resolve. Unfortunately, emergency surgery cannot be postponed in some situations, and in these cases the magnitude of intraperitoneal

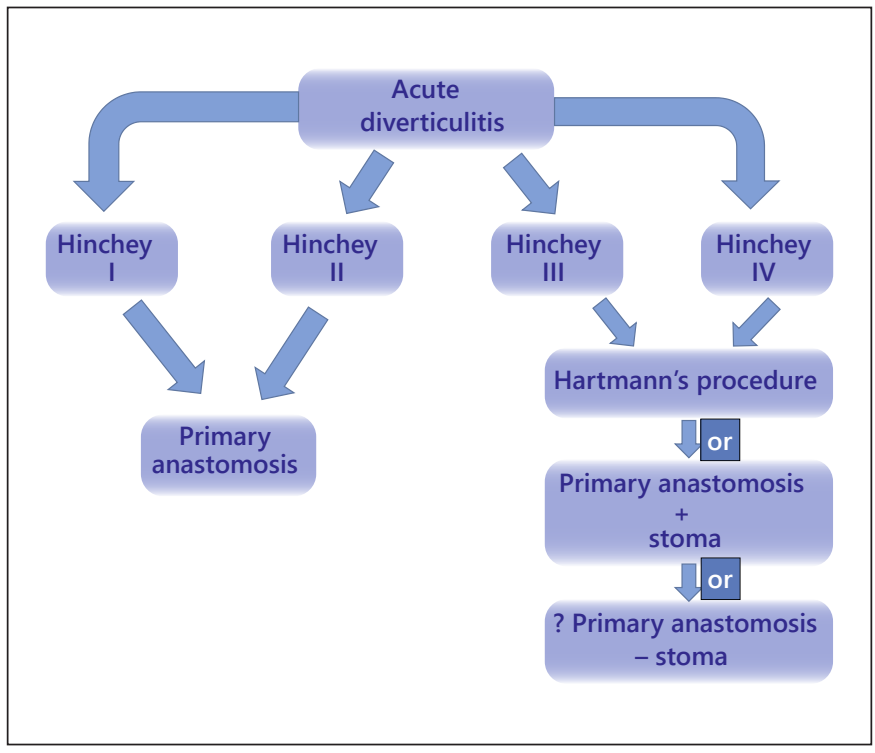

Fig. 1. Algorithm of surgical procedures as per Hinchey classification.

contamination dictates the choice of reconstruction. The Hinchey classification is widely used to assess the degree of contamination. Grade I is used for a small confined pericolic or mesenteric abscess. Grade II is for a larger abscess often confined to the pelvis. Grade III is for perforated diverticulitis, ruptured abscess, and/or purulent peritonitis. Last, grade IV is for ruptured diverticulitis with fecal peritonitis. Generally, with localized contamination - grades I and II - primary anastomosis without a protective ostomy can be performed, while with more diffuse contamination - grades III and IV - a two-staged procedure is generally preferred. The two-staged procedures are either a Hartmann's procedure or a primary anastomosis with a protective proximal diverting stoma (Fig. 1).

Sixty-two patients with acute left-sided colonic Hinchey III and IV from 4 centers were randomized to Hartmann's procedure or to primary anastomosis with diverting ileostomy. The overall complication rate for both resection and stoma reversal operations was comparable. The stoma reversal rate after primary anastomosis with diverting ileostomy was higher. Serious complications, operating time, hospital stay, and in-hospital costs were significantly reduced in the primary anastomosis group [45].

Similarly, 102 patients with Hinchey grade III or IV diverticulitis randomly assigned to primary anastomosis or Hartmann's procedure were studied in the DIVERTI
Al Harakeh/Paily/Doughan/Shaikh 
French trial. The difference in the mortality and morbidity rates was insignificant. However, at 18 months of follow-up the primary anastomosis patients were more likely to revert their stomas (96 vs. 65\%). Of note, receiving a protective stoma was at the discretion of the surgeon, whereby two-thirds of patients in the primary anastomosis group ended up receiving a protective stoma and onethird did not. Morbidity in the stoma subgroup was higher, but this result was likely biased since, in the subgroup that did not receive a stoma, all but one had grade III Hinchey diverticulitis [46].

\section{Surgical Margins}

Surgical margins have been investigated as well and it was found that total sigmoid colectomy with rectal anastomosis was associated with lower recurrence $[3,47]$. In fact, Thaler et al. [47] found that colocolonic anastomosis with preservation of the distal sigmoid colon had a 4 -fold risk of recurrent diverticulitis compared with colorectal anastomosis. Because the transverse and descending colon hardly ever have recurrence, it is unnecessary to resect all the diverticula-containing colon and thus any proximal soft and nonedematous colon segment is generally acceptable as a proximal margin [3]. On the contrary, the extent of resection and type of anastomosis were not important factors for recurrence in the study by Andeweg et al. [38].

Open surgery versus laparoscopic approach was assessed in a meta-analysis of 19 nonrandomized studies. The meta-analysis included 1,014 patients in the elective laparoscopic resection arm and 1,369 patients in the open arm. Open surgery was associated with significantly higher rates of wound infection, blood transfusion, postoperative ileus, and incisional hernia. This difference is highlighted in the short-term outcomes; however, longterm outcomes are comparable [48].

In the Sigma trial that included 104 patients with Hinchey I and II diverticulitis, laparoscopic approach was associated with longer operating time but less pain, improved quality of life, shorter hospital stays, and 15.4\% reduction in major complication rates. The conversion rate was $19.2 \%$, and the mortality rate was $1 \%$ [49]. However, in 2017 a Cochrane review that included this study found no superiority of one technique over the other [50].

Bowel obstruction, peritonitis, sepsis, and fistula were complications independently associated with mortality if they occurred after the first episode of diverticulitis. In addition, elective surgery carried a considerably lower mortality rate compared to emergency surgery for the re-

Recurrent Acute Diverticulitis: When to Operate? current episode. Recurrent attacks of acute diverticulitis carry the risk of gradual scarring and fibrosis with the sequela of forming a stricture.

Diverticular disease rarely causes complete obstruction. This allows either bowel preparation or on-table lavage to be done and in turn permits primary anastomosis. On the other hand, in their systematic review, Jones et al. [34] found that endoluminal self-expanding stents caused more cases of perforation, stent migration, and recurrent obstruction in benign colorectal obstructions compared to stenting malignant cases. However, these stents when used as a bridge to elective surgery avoided stoma in $43 \%$ of patients with diverticulitis [51].

\section{Special Consideration}

Immunosuppressed patients are especially predisposed to develop acute diverticulitis $(0.02$ vs. $1 \%)$ and, if they do, have a higher necessity to undergo emergency surgery (10-25 vs. $40 \%)$. Moreover, they have a higher mortality should they be operated on in an emergency ( 5 vs. 30\%) [52, 53]. Furthermore, because of their immunosuppressed state, these patients often have atypical presentation and diagnosis is often delayed; therefore, they usually have a more severe acute diverticulitis and higher likelihood of perforation [54]. Hence, many surgeons offer elective surgery after resolution of the first attack of diverticulitis. However, these patients are often not cleared for surgical intervention and, therefore, recommendations on surgical intervention should be individualized based on their general health status. Concerning HIV-positive patients, lower CD4+ counts carry a poorer prognosis after surgery [54].

\section{Conclusion}

Studies in the literature have shown a highly variable approach in managing this group of patients. There is no well-defined evidence-based protocol that includes indication, timing, and method of surgery. Individualizing cases is the trend in dealing with these patients. Weighing the risk of developing chronic pain from conservative management versus the low probability of needing surgical intervention together with the advent of dependence on procedures performed by interventional radiologists to treat complicated diverticulitis is essential in gearing towards a certain treatment modality. Special attention should be paid to patients who are young and of female gender as they carry a higher risk of recurrence, in addition to the group of immunosuppressed patients who are 
still highly preferred to undergo elective surgical intervention to prevent the unfortunate complications which remain obscure until advanced stages in this subgroup of patients. Multicenter randomized controlled trials are needed to match the most beneficial treatment modalities with each patient subgroup.

\section{Disclosure Statement}

The authors have no disclosures.

\section{Author Contributions}

All authors contributed equally to the paper.

\section{References}

1 Poletti PA, Platon A, Rutschmann O, Kinkel K, Nyikus V, Ghiorghiu S, et al. Acute left colonic diverticulitis: can CT findings be used to predict recurrence? AJR Am J Roentgenol. 2004 May;182(5):1159-65.

2 Anaya DA, Flum DR. Risk of emergency colectomy and colostomy in patients with diverticular disease. Arch Surg. 2005 Jul;140(7): 681-5.

3 Benn PL, Wolff BG, Ilstrup DM. Level of anastomosis and recurrent colonic diverticulitis. Am J Surg. 1986 Feb;151(2):269-71.

4 Bharucha AE, Parthasarathy G, Ditah I, Fletcher JG, Ewelukwa O, Pendlimari R, et al. Temporal Trends in the Incidence and Natural History of Diverticulitis: A PopulationBased Study. Am J Gastroenterol. 2015 Nov; 110(11):1589-96.

5 Eglinton T, Nguyen T, Raniga S, Dixon L, Dobbs B, Frizelle FA. Patterns of recurrence in patients with acute diverticulitis. Br J Surg. 2010 Jun;97(6):952-7.

6 El-Sayed C, Radley S, Mytton J, Evison F, Ward ST. Risk of Recurrent Disease and Surgery Following an Admission for Acute Diverticulitis. Dis Colon Rectum. 2018 Mar; 61(3):382-9.

7 Hall JF, Roberts PL, Ricciardi R, Read T, Scheirey C, Wald C, et al. Long-term followup after an initial episode of diverticulitis: what are the predictors of recurrence? Dis Colon Rectum. 2011 Mar;54(3):283-8.

8 Li D, de Mestral C, Baxter NN, McLeod RS, Moineddin R, Wilton AS, et al. Risk of readmission and emergency surgery following nonoperative management of colonic diverticulitis: a population-based analysis. Ann Surg. 2014 Sep;260(3):423-30; discussion 430-1.

9 Ritz JP, Lehmann KS, Frericks B, Stroux A, Buhr HJ, Holmer C. Outcome of patients with acute sigmoid diverticulitis: multivariate analysis of risk factors for free perforation. Surgery. 2011 May;149(5):606-13.

10 Wolff BG, Ready RL, MacCarty RL, Dozois $\mathrm{RR}$, Beart RW Jr. Influence of sigmoid resection on progression of diverticular disease of the colon. Dis Colon Rectum. 1984 Oct; 27(10):645-7.

11 van de Wall BJ, Stam MA, Draaisma WA, Stellato R, Bemelman WA, Boermeester MA, et al.; DIRECT trial collaborators. Surgery versus conservative management for recur- rent and ongoing left-sided diverticulitis (DIRECT trial): an open-label, multicentre, randomised controlled trial. Lancet Gastroenterol Hepatol. 2017 Jan;2(1):13-22.

12 Peppas G, Bliziotis IA, Oikonomaki D, Falagas ME. Outcomes after medical and surgical treatment of diverticulitis: a systematic review of the available evidence. J Gastroenterol Hepatol. 2007 Sep;22(9):1360-8.

13 Andeweg CS, Berg R, Staal JB, ten Broek RP, van Goor H. Patient-reported Outcomes After Conservative or Surgical Management of Recurrent and Chronic Complaints of Diverticulitis: Systematic Review and Meta-analysis. Clin Gastroenterol Hepatol. 2016 Feb; 14(2):183-90

14 Hupfeld L, Pommergaard HC, Burcharth J, Rosenberg J. Emergency admissions for complicated colonic diverticulitis are increasing: a nationwide register-based cohort study. Int J Colorectal Dis. 2018 Jul;33(7):879-86.

15 Katz LH, Guy DD, Lahat A, Gafter-Gvili A, Bar-Meir S. Diverticulitis in the young is not more aggressive than in the elderly, but it tends to recur more often: systematic review and meta-analysis. J Gastroenterol Hepatol. 2013 Aug;28(8):1274-81.

16 Rafferty J, Shellito P, Hyman NH, Buie WD; Standards Committee of American Society of Colon and Rectal Surgeons. Practice parameters for sigmoid diverticulitis. Dis Colon Rectum. 2006 Jul;49(7):939-44.

17 Liu PH, Cao Y, Keeley BR, Tam I, Wu K, Strate LL, et al. Adherence to a Healthy Lifestyle is Associated With a Lower Risk of Diverticulitis among Men. Am J Gastroenterol. 2017 Dec;112(12):1868-76.

18 Strate LL, Liu YL, Syngal S, Aldoori WH, Giovannucci EL. Nut, corn, and popcorn consumption and the incidence of diverticular disease. JAMA. 2008 Aug; 300(8): 907-14.

19 Aldoori WH, Giovannucci EL, Rimm EB, Wing AL, Trichopoulos DV, Willett WC. A prospective study of alcohol, smoking, caffeine, and the risk of symptomatic diverticular disease in men. Ann Epidemiol. 1995 May; 5(3):221-8.

20 Strate LL, Liu YL, Huang ES, Giovannucci EL, Chan AT. Use of aspirin or nonsteroidal antiinflammatory drugs increases risk for diverticulitis and diverticular bleeding. Gastroenterology. 2011 May;140(5):1427-33.
21 Maguire LH, Song M, Strate LE, Giovannucci EL, Chan AT. Higher serum levels of vitamin $\mathrm{D}$ are associated with a reduced risk of diverticulitis. Clin Gastroenterol Hepatol. 2013 Dec;11(12):1631-5.

22 Shahedi K, Fuller G, Bolus R, Cohen E, Vu M, Shah R, et al. Long-term risk of acute diverticulitis among patients with incidental diverticulosis found during colonoscopy. Clin Gastroenterol Hepatol. 2013 Dec;11(12):1609-13.

23 Laméris W, van Randen A, Bipat S, Bossuyt PM, Boermeester MA, Stoker J. Graded compression ultrasonography and computed tomography in acute colonic diverticulitis: meta-analysis of test accuracy. Eur Radiol. 2008 Nov; 18(11):2498-511.

24 Andeweg CS, Felt-Bersma R, Verbon A, Stoker J, Boermeester M, Bleichrodt R. [Summary of the practice guideline on diverticulitis in the colon: diagnostics and treatment in specialty care]. Ned Tijdschr Geneeskd. 2013; 157(15):A6124. Dutch.

25 Horgan AF, McConnell EJ, Wolff BG, The S, Paterson C. Atypical diverticular disease: surgical results. Dis Colon Rectum. 2001 Sep; 44(9):1315-8.

26 Clemens CH, Samsom M, Roelofs J, van Berge Henegouwen GP, Smout AJ. Colorectal visceral perception in diverticular disease. Gut. 2004 May;53(5):717-22.

27 Tursi A, Brandimarte G, Giorgetti GM, Elisei W. Assessment of small intestinal bacterial overgrowth in uncomplicated acute diverticulitis of the colon. World J Gastroenterol. 2005 May;11(18):2773-6.

28 Cohen E, Fuller G, Bolus R, Modi R, Vu M, Shahedi $\mathrm{K}$, et al. Increased risk for irritable bowel syndrome after acute diverticulitis. Clin Gastroenterol Hepatol. 2013 Dec;11(12): 1614-9.

29 Ricciardi R, Baxter NN, Read TE, Marcello PW, Hall J, Roberts PL. Is the decline in the surgical treatment for diverticulitis associated with an increase in complicated diverticulitis? Dis Colon Rectum. 2009 Sep;52(9):1558-63.

30 Janes S, Meagher A, Frizelle FA. Elective surgery after acute diverticulitis. Br J Surg. 2005 Feb;92(2):133-42.

31 Stollman N, Magowan S, Shanahan F, Quigley EM; DIVA Investigator Group. A randomized controlled study of mesalamine after acute diverticulitis: results of the DIVA trial. J Clin Gastroenterol. 2013 Aug;47(7):621-9. 
32 Raskin JB, Kamm MA, Jamal MM, Márquez J, Melzer E, Schoen RE, et al. Mesalamine did not prevent recurrent diverticulitis in phase 3 controlled trials. Gastroenterology. 2014 Oct; 147(4):793-802.

33 Carter F, Alsayb M, Marshall JK, Yuan Y. Mesalamine (5-ASA) for the prevention of recurrent diverticulitis. Cochrane Database Syst Rev. 2017 Oct;10:CD009839.

34 Jones OM, Stevenson AR, Clark D, Stitz RW Lumley JW. Laparoscopic resection for diverticular disease: follow-up of 500 consecutive patients. Ann Surg. 2008 Dec;248(6):1092-7.

35 Royds J, O'Riordan JM, Eguare E, O'Riordan D, Neary PC. Laparoscopic surgery for complicated diverticular disease: a single-centre experience. Colorectal Dis. 2012 oct;14(10): 1248-54.

36 Jonas WB, Crawford C, Colloca L, Kaptchuk TJ, Moseley B, Miller FG, et al. To what extent are surgery and invasive procedures effective beyond a placebo response? A systematic review with meta-analysis of randomised, sham controlled trials. BMJ Open. 2015 Dec; 5(12): 0009655.

37 Salem TA, Molloy RG, O’Dwyer PJ. Prospective, five-year follow-up study of patients with symptomatic uncomplicated diverticular disease. Dis Colon Rectum. 2007 Sep;50(9): $1460-4$.

38 Andeweg C, Peters J, Bleichrodt R, van Goor $H$. Incidence and risk factors of recurrence after surgery for pathology-proven diverticular disease. World J Surg. 2008 Jul;32(7):1501-6.

39 Chapman JR, Dozois EJ, Wolff BG, Gullerud RE, Larson DR. Diverticulitis: a progressive disease? Do multiple recurrences predict less favorable outcomes? Ann Surg. 2006 Jun; 243(6):876-880; discussion 880-3.
40 Klarenbeek BR, Samuels M, van der Wal MA, van der Peet DL, Meijerink WJ, Cuesta MA. Indications for elective sigmoid resection in diverticular disease. Ann Surg. 2010 Apr; 251(4):670-4

41 Chautems RC, Ambrosetti P, Ludwig A, Mermillod B, Morel P, Soravia C. Long-term follow-up after first acute episode of sigmoid diverticulitis: is surgery mandatory?: a prospective study of 118 patients. Dis Colon Rectum. 2002 Jul;45(7):962-6.

42 Morris AM, Regenbogen SE, Hardiman KM, Hendren S. Sigmoid diverticulitis: a systematic review. JAMA. 2014 Jan;311(3):287-97.

43 Boostrom SY, Wolff BG, Cima RR, Merchea A, Dozois EJ, Larson DW. Uncomplicated diverticulitis, more complicated than we thought. J Gastrointest Surg. 2012 Sep;16(9): 1744-9.

44 Bendl RF, Bergamaschi R. Do Patients Mandate Resection After a First Episode of Acute Diverticulitis of the Colon with a Complication? Adv Surg. 2017 Sep;51(1):179-91.

45 Oberkofler CE, Rickenbacher A, Raptis DA, Lehmann K, Villiger P, Buchli C, et al. A multicenter randomized clinical trial of primary anastomosis or Hartmann's procedure for perforated left colonic diverticulitis with purulent or fecal peritonitis. Ann Surg. 2012 Nov;256(5):819-26; discussion 826-7.

46 Bridoux V, Regimbeau JM, Ouaissi M, Mathonnet M, Mauvais F, Houivet E, et al. Hartmann's Procedure or Primary Anastomosis for Generalized Peritonitis due to Perforated Diverticulitis: A Prospective Multicenter Randomized Trial (DIVERTI). J Am Coll Surg. 2017 Dec;225(6):798-805.
47 Thaler K, Baig MK, Berho M, Weiss EG, Nogueras JJ, Arnaud JP, et al. Determinants of recurrence after sigmoid resection for uncomplicated diverticulitis. Dis Colon Rectum. 2003 Mar;46(3):385-8.

48 Siddiqui MR, Sajid MS, Qureshi S, Cheek E Baig MK. Elective laparoscopic sigmoid resection for diverticular disease has fewer complications than conventional surgery: a metaanalysis. Am J Surg. 2010 Jul;200(1):144-61.

49 Klarenbeek BR, Veenhof AA, Bergamaschi R, van der Peet DL, van den Broek WT, de Lange ES, et al. Laparoscopic sigmoid resection for diverticulitis decreases major morbidity rates: a randomized control trial: short-term results of the Sigma Trial. Ann Surg. 2009 Jan;249(1): 39-44.

50 Abraha I, Binda GA, Montedori A, Arezzo A, Cirocchi R. Laparoscopic versus open resection for sigmoid diverticulitis. Cochrane $\mathrm{Da}$ tabase Syst Rev. 2017 Nov;11:CD009277.

51 Currie A, Christmas C, Aldean H, Mobasheri M, Bloom IT. Systematic review of self-expanding stents in the management of benign colorectal obstruction. Colorectal Dis. 2014 Apr;16(4):239-45

52 Biondo S, Trenti L, Elvira J, Golda T, Kreisler E. Outcomes of colonic diverticulitis according to the reason of immunosuppression. Am J Surg. 2016 Sep;212(3):384-90.

53 Rose J, Parina RP, Faiz O, Chang DC, Talamini MA. Long-term Outcomes After Initial Presentation of Diverticulitis. Ann Surg. 2015 Dec;262(6):1046-53.

54 Chapman J, Davies M, Wolff B, Dozois E, Tessier D, Harrington J, et al. Complicated diverticulitis: is it time to rethink the rules? Ann Surg. 2005 Oct;242(4):576-81; discussion 581-3. 